\title{
Essay for the CIHR/CMAJ award: impact of the Perioperative Ischemic Evaluation (POISE) trial
}

\author{
Philip J. Devereaux MD PhD, Gordon Guyatt MD MSc, Homer Yang MD, Salim Yusuf MD DPhil; for the \\ POISE-1 investigators
}

See related articles by Kelsall at www.cmaj.ca/cgi/doi/10.1503/cmaj.110255, by Hull at www.cmaj.ca/cgi/doi/10.1503/cmaj.110364, and by Frank and colleagues at www.cmaj.ca/cgi/doi/10.1503/cmaj.110358.

Russell Hull, the POISE-1 investigators, and Cyril Frank and colleagues for the Alberta Bone and Joint Health Institute are the highest-ranking winners of the 2009-2010 CIHR/CMAJ competition for the Top Achievements in Health Research. Dr. Devereaux and colleagues describe the POISE trial in the following essay. Dr. Hull's essay, the essay by Dr. Frank and colleagues, and synopses of the other three winning achievements are available at www.cmaj.ca.

$\mathrm{D}$ uring the last few decades, substantial advances in noncardiac surgery have improved the treatment of disease and the quality of life for patients. As a result, the number of patients undergoing noncardiac surgery is growing. A recent study that used surgical data from 56 countries suggests that 200 million major noncardiac surgical procedures are undertaken annually around the world.'

Noncardiac surgery is associated with major vascular complications (i.e., vascular death, nonfatal myocardial infarction (MI), nonfatal cardiac arrest, and nonfatal stroke). Worldwide each year, 3-5 million adult patients have a perioperative vascular complication in the first 30 days after surgery, ${ }^{2}$ a number similar to the annual global incidence of new patients acquiring HIV. ${ }^{3}$

Despite the magnitude of this public health problem, there has existed a paucity of adequately powered randomized controlled trials that have evaluated interventions that may lower the risk of major perioperative cardiovascular events. Because of this gap, a team of Canadian investigators led a large international randomized controlled trial evaluating the effects of a $B$-blocker in patients undergoing noncardiac surgery. This trial was called the Perioperative Ischemic Evaluation (POISE) trial, and the primary results from this trial received fast-track publication in the Lancet in May, 2008. ${ }^{4}$

\section{Rationale for the POISE trial}

Two small randomized controlled trials (312 patients in total), which were published in the 1990s and had important methodologic limitations (i.e., they were unblinded, they were stopped early for unexpectedly large treatment effect in the setting of few events, and there was a failure to follow the intention-to-treat principle), suggested that $\beta$-blockers given around the time of noncardiac surgery resulted in a $90 \%$ relative risk reduction in the composite outcome of cardiovascular death and nonfatal MI at the 30-day follow-up and a 55\% relative risk reduction in death at the two-year follow-up. ${ }^{5,6}$ Primarily based on the results of these two trials, the American College of Physicians and the American College of Cardiology/American Heart Association perioperative guidelines recommended giving a $\beta$-blocker to at-risk patients undergoing noncardiac surgery. ${ }^{7.8}$ More recently, two moderately sized randomized controlled trials (1417 patients in total), which avoided the methodological limitations of the previous trials, did not show a benefit with perioperative $\beta$-blockers. ${ }^{9}{ }^{10}$ Despite those findings, guideline committees continued to
Competing interests: Funding in the form of a grant to P.J. Devereaux was received from AstraZeneca and was used to purchase the drug studied in the POISE Trial. Salim Yusuf has received grants and financial support from AstraZeneca. No other competing interests were declared.

Correspondence to: Dr. P.J. Devereaux, philipj@mcmaster.ca

CMAJ 2011. DOI:10.1503 /cmaj.110292

\section{KEY POINTS}

- Worldwide, over 200 million adults have major noncardiac surgery each year, and several million experience a major vascular complication (i.e., vascular death, nonfatal myocardial infarction, nonfatal cardiac arrest or nonfatal stroke).

- For over a decade, guideline committees had recommended that at-risk patients receive a perioperative $\beta$-blocker based on the results of two small trials with methodologic limitations.

- The POISE Trial, led by Canadian investigators, included 8351 patients from 190 centres in 23 countries; the trial showed that, although metoprolol CR prevented perioperative myocardial infarction, it increased the 30-day risk of total mortality and stroke.

- POISE has shown how perioperative $\beta$-blockers may increase the risk of death and stroke at 30-day follow-up by increasing the risk of clinically significant hypotension. 
recommend that physicians give $\beta$-blockers to patients undergoing noncardiac surgery. ${ }^{11}$

\section{Description of POISE}

The POISE trial included 8351 patients from 190 hospitals in 23 countries, and $99.8 \%$ of the patients completed the 30-day follow-up. Patients, health care providers, data collectors and outcome adjudicators were blind to treatment allocation, and all analyses followed the intention-to-treat principle. Patients were randomized to treatment with extended-release metoprolol succinate (metoprolol CR) or placebo starting two to four hours before surgery and continuing for 30 days. The goal was to administer $200 \mathrm{mg}$ of the study drug (i.e., metoprolol CR or matching placebo) daily, but patients had to have a systolic blood pressure greater than or equal to $100 \mathrm{~mm} \mathrm{Hg}$ and a heart rate greater than or equal to 50 beats/min to receive the study drug. ${ }^{12}$

At the 30-day follow-up, 244 (5.8\%) of the patients taking metoprolol and $290(6.9 \%)$ of the patients given a placebo had experienced the primary outcome - a composite of cardiovascular death, nonfatal MI and nonfatal cardiac arrest (hazard ratio [HR] $0.84,95 \%$ confidence interval [CI] $0.70-0.99, p=0.04)$. This benefit was due to a reduction in the number of patients with $\mathrm{MI}$ (176 [4.2\%] in the metoprolol group compared with 239 [5.7\%] in the placebo group; HR 0.73 , $95 \%$ CI $0.60-0.89, p=0.002)$. In contrast to this benefit, mortality was higher in the metoprolol group (129 [3.1\%] patients) than in the placebo group (97 [2.3\%] patients) (HR 1.33, 95\% CI $1.03-1.74, p=0.03)$. More strokes also occurred in the metoprolol group (41 [1.0\%] patients) than in the placebo group (19 [0.5\%] patients) (HR $2.17,95 \%$ CI 1.26-3.74, $p=0.005)$.

Although the POISE trial was an international effort, the Canadian investigators (a multidisciplinary group of anesthesiologists, surgeons and specialists in internal medicine) were responsible for originating the idea for the trial, conducting the POISE pilot study, writing the POISE protocol, obtaining funding for the trial through a grant from the Canadian Institutes of Health Research (CIHR), developing the international collaboration of 190 centres in 23 countries, recruiting 3548 of the 8351 patients, doing the analyses, and writing the papers describing the POISE methods and main results. (All of the Canadian investigators involved in the POISE Trial are listed in Appendix 1, available at www.cmaj.ca /cgi/content/full/cmaj.110292/DC1.) The Population Health Research Institute, McMaster University and Hamilton Health Sciences coordinated the study and managed the data under the supervision of the operations committee.

\section{What we learned from POISE}

POISE has provided many important insights into vascular complications among patients undergoing noncardiac surgery. POISE was the first trial to demonstrate a statistically significant increase in death and stroke with a perioperative B-blocker. These results almost did not come to light because the POISE investigators encountered substantial opposition to this trial from many physicians who believed that perioperative $\beta$-blockers were beneficial and without any substantial risks. ${ }^{13}$

POISE analyses have shown how perioperative B-blockers may increase the risk of death and stroke. POISE has demonstrated that more patients in the metoprolol group (625 patients [15.0\%]) than in the placebo group (404 patients [9.7\%]) experienced clinically significant hypotension (i.e., systolic blood pressure $<90 \mathrm{~mm} \mathrm{Hg}$ and requiring medical intervention; 95\% CI 1.38-1.74, $p<$ $0.0001)$. Similarly, clinically significant bradycardia (i.e., bradycardia requiring medical intervention) was more common in the metoprolol group (277 [6.6\%] patients) than in the placebo group (101 [2.4\%] patients) (HR 2.74, 95\% CI 2.19$3.43, p<0.0001)$.

Post-hoc multivariable analyses were done to determine population attributable risks, which represent the proportion of all outcomes attributable to the relevant risk factor if causality were proven. Clinically significant hypotension had the largest population attributable risk $(37.3 \%)$ for death and the largest intraoperative/postoperative population attributable risk (14.7\%) for stroke. Furthermore, for the outcome of death, the population attributable risk associated with perioperative stroke was $8.0 \%$; with clinically significant bradycardia, the risk was $7.9 \%$. Therefore, perioperative clinically significant hypotension, bradycardia and stroke caused by perioperative treatment with a $ß$-blocker potentially accounted for over $50 \%$ of the deaths seen during the POISE trial. Although clinically significant hypotension was an important predictor of perioperative stroke, our analysis explained fewer than half of the strokes. It is possible that more hypotension occurred that was clinically relevant but that did not fulfill our definition, and that this hypotension contributed to the higher incidence of stroke.

Two-thirds of the POISE patients who had a perioperative MI did not experience ischemic symptoms. We believe this occurred because about $75 \%$ of the MIs happened during the first 48 hours after surgery when most patients are receiving high doses of analgesic medications. The MIs without ischemic symptoms were independent predictors of death at the 30-day follow-up (HR 3.45, 95\% CI 2.20-5.41). This finding shows the benefit of moni- 
toring troponins (part of the POISE protocol) for the first three days after surgery.

\section{Future directions}

POISE has resulted in several new studies that are being led by the Canadian POISE investigators. The Vascular Events In Noncardiac Surgery Patients Cohort Evaluation (VISION) Study is a prospective cohort study of 40000 patients undergoing noncardiac surgery in several countries around the world, and more than 20000 participants have already been recruited (for further information, please see www.clinicaltrials.gov, identifier no. NCT00512109). Observations from POISE provided the bases for the following VISION objectives: to determine the incidence of major vascular complications among a representative sample of adults undergoing noncardiac surgery (in POISE, the event rate was three times higher than had been predicted in previous studies); to develop a clinical model to predict major perioperative vascular complications (in POISE, the previous models underestimated risk); to determine the proportion of perioperative MIs that would probably go unrecognized without perioperative troponin monitoring; and to determine if these MIs independently influence a patient's risk of vascular death during the first year after surgery. VISION also involves many substudies, including a study of the effects of perioperative hypotension on major vascular complications and a biobank study that will provide insights into the pathophysiology, prediction and identification of perioperative MIs.

The POISE-2 trial is a factorial design (lowdose acetylsalicylic acid [ASA] v. placebo and low-dose clonidine v. placebo) that builds on the findings of POISE. We have completed the POISE-2 pilot study and have already randomized 650 of the 10000 patients into the trial. Observational data from POISE showed that ASA was the perioperative drug associated with the largest statistically significant reduction in death. Clonidine lowers perioperative heart rates, which appeared to be a primary mechanism of how metoprolol CR prevented MIs in POISE. In the randomized controlled trials done to date, perioperative low-dose clonidine is associated with less clinically significant hypotension than was seen in the perioperative $\beta$-blocker trials. ${ }^{14-16}$

POISE showed that a perioperative $\beta$-blocker results in both benefits and risks. Building on this knowledge, we have initiated POISE-2; hopefully, through low-dose clonidine, we can establish a way to obtain the benefits we saw in POISE while mitigating the risks. The knowledge gained through these trials will improve the safety of surgery for the 200 million adults worldwide who annually undergo noncardiac procedures.

\section{References}

1. Weiser TG, Regenbogen SE, Thompson KD, et al. An estimation of the global volume of surgery: a modelling strategy based on available data. Lancet 2008;372:139-44

2. Devereaux PJ, Chan M, Eikelboom J. Major vascular complications in patients undergoing noncardiac surgery: magnitude of the problem, risk prediction, surveillance, and prevention. In: Yusuf S, Cairns JA, Camm AJ, et al., editors. Evidence based cardiology. 3rd ed. West Sussex (UK): Wiley-Blackwell; 2010. p. 47-62.

3. Centers for Disease Control and Prevention (CDC). The Global HIV/AIDS pandemic, 2006. MMWR Morb Mortal Wkly Rep 2006;55:841-4.

4. Devereaux PJ, Yang H, Yusuf S, et al. Effects of extendedrelease metoprolol succinate in patients undergoing non-cardiac surgery (POISE trial): a randomised controlled trial. Lancet 2008;371:1839-47.

5. Mangano DT, Layug EL, Wallace A, et al. Effect of atenolol on mortality and cardiovascular morbidity after noncardiac surgery Multicenter Study of Perioperative Ischemia Research Group. N Engl J Med 1996;335:1713-20.

6. Poldermans D, Boersma E, Bax JJ, et al. The effect of bisoprolol on perioperative mortality and myocardial infarction in high-risk patients undergoing vascular surgery. Dutch Echocardiographic Cardiac Risk Evaluation Applying Stress Echocardiography Study Group. N Engl J Med 1999;341:1789-94.

7. Palda VA, Detsky AS. Perioperative assessment and management of risk from coronary artery disease. Ann Intern Med 1997;127:313-28.

8. Eagle KA, Berger PB, Calkins $\mathrm{H}$, et al. ACC/AHA guideline update for perioperative cardiovascular evaluation for noncardiac surgery - executive summary a report of the American College of Cardiology/American Heart Association Task Force on Practice Guidelines (Committee to Update the 1996 Guidelines on Perioperative Cardiovascular Evaluation for Noncardiac Surgery). Circulation 2002;105:1257-67.

9. Yang H, Raymer K, Butler R, et al. The effects of perioperative beta-blockade: results of the Metoprolol after Vascular Surgery (MaVS) study, a randomized controlled trial. Am Heart J 2006; 152:983-90.

10. Juul AB, Wetterslev J, Gluud C, et al. Effect of perioperative beta blockade in patients with diabetes undergoing major noncardiac surgery: randomised placebo controlled, blinded multicentre trial. BMJ 2006;332:1482.

11. Fleisher LA, Beckman JA, Brown KA, et al. ACC/AHA 2007 Guidelines on perioperative cardiovascular evaluation and care for noncardiac surgery: Executive summary: a report of the American College of Cardiology/American Heart Association Task Force on Practice Guidelines (Writing Committee to Revise the 2002 Guidelines on Perioperative Cardiovascular Evaluation for Noncardiac Surgery) developed in collaboration with the American Society of Echocardiography, American Society of Nuclear Cardiology, Heart Rhythm Society, Society of Cardiovascular Anesthesiologists, Society for Cardiovascular Angiography and Interventions, Society for Vascular Medicine and Biology, and Society for Vascular Surgery. [published erratum in J Am Coll Cardiol 2008;52:794-7.]. J Am Coll Cardiol 2007;50:1707-32.

12. Devereaux PJ, Yang H, Guyatt GH, et al. Rationale, design, and organization of the perioperative ischemic evaluation (POISE) trial: a randomized controlled trial of metoprolol versus placebo in patients undergoing noncardiac surgery. Am Heart J 2006; 152:223-30.

13. London MJ. Quo vadis, perioperative beta blockade? Are you "POISE'd" on the brink? Anesth Analg 2008;106:1025-30.

14. Wallace AW, Galindez D, Salahieh A, et al. Effect of clonidine on cardiovascular morbidity and mortality after noncardiac surgery. Anesthesiology 2004;101:284-93.

15. Ellis JE, Drijvers G, Pedlow S, et al. Premedication with oral and transdermal clonidine provides safe and efficacious postoperative sympatholysis. Anesth Analg 1994;79:1133-40.

16. Devereaux PJ, Beattie WS, Choi PT, et al. How strong is the evidence for the use of perioperative beta blockers in noncardiac surgery? Systematic review and meta-analysis of randomised controlled trials. BMJ 2005;331:313-21.

Affiliations: From the Departments of Medicine and Clinical Epidemiology and Biostatistics (Devereaux, Yusuf, Guyatt), McMaster University, Hamilton, Ont.; Population Health Research Institute (Devereaux, Yusuf), McMaster University, Hamilton, Ont.; the Department of Anaesthesia (Yang), University of Ottawa, Ottawa, Ont. 